\title{
Novel BRAF fusions in pediatric histiocytic neoplasms define distinct therapeutic responsiveness to RAF paradox breakers
}

Payal Jain ${ }^{1 *}$, Lea F. Surrey ${ }^{2 *}$, Joshua Straka ${ }^{1}$, Pierre Russo ${ }^{2}$, Richard Womer ${ }^{3}$, Marilyn M. Li ${ }^{2}$, Phillip B. Storm ${ }^{1,4,5}$, Angela Waanders ${ }^{6}$, Michael D. Hogarty ${ }^{3}$, Adam Resnick $^{1 * *}$, Jennifer Picarsic $^{7 * *}$

1. Center for Data Driven Discovery in Biomedicine (D3B), Children's Hospital of Philadelphia, Philadelphia, PA USA

2. Department of Pathology and Laboratory Medicine, Children's Hospital of Philadelphia, Philadelphia, PA USA

3. Department of Pediatrics, Division of Hematology and Oncology, Children's Hospital of Philadelphia (CHOP), Philadelphia, PA USA

4. Department of Neurosurgery, University of Pennsylvania, Philadelphia, PA, USA

5. Division of Neurosurgery, The Children's Hospital of Philadelphia, PA, USA

6. Department of Pediatrics, Feinberg School of Medicine Northwestern University, Chicago, IL, USA

7. Department of Pathology, University of Pittsburgh School of Medicine Pittsburgh, PA USA /Department of Pathology, Cincinnati Children's Hospital Medical Center

*Both authors contributed equally as first authors

**Both authors contributed equally as senior authors

Running Title: Novel BRAF fusions in pediatric histiocytic tumors

Histiocytic neoplasms are a diverse group of clonal hematopoietic disorders that are driven by mutations activating the mitogen-activated protein kinase (MAPK) and phosphoinositide 3kinases (PI3K) pathways ${ }^{1-3}$. While BRAF-V600E is the most common alteration in histiocytic neoplasms, multiple alternate pathway activating mechanisms have been described, including MAP2K1, ARAF, PIK3CA, NRAS, and KRAS mutations as well as BRAF, ALK, and NTRK1 fusions ${ }^{1-3}$. BRAF-fusions previously reported in cases of histiocytic neoplasms ${ }^{1,4-7}$ were found to contain the $\mathrm{N}$-terminal region of another gene (often of unclear significance) joined to the BRAF kinase domain (including exons 9-18 or 11-18), resulting in the loss of the BRAF N-terminal regulatory RAS-binding regions (exons 1-8). Despite the prevalence of BRAF-alterations in histiocytic tumors, to date there have been no detailed molecular investigations comparing BRAF-fusions found in distinct sub-types of histiocytic neoplasms and only one study explored responsiveness of such BRAF-fusions to single-agent RAF-therapies ${ }^{4}$. To address this, we present two pediatric histiocytosis cases with divergent pathologic and clinical features, each harboring a novel BRAFfusion as identified by a clinically validated next-generation sequencing panel (Supplemental Methods and Table 1) and study their in vitro responsiveness to RAF-targeted inhibitors. 
Case 1 , a 16 year-old female with a $2.5 \mathrm{~cm}$ rapidly growing subcutaneous thigh mass was diagnosed with a malignant histiocytic neoplasm ("M group") ${ }^{8}$, with a phenotype spanning histiocytic sarcoma (CD163/CD14/CD68+) and Langerhans cell sarcoma (CD1a/Langerin/S100) with a modestly elevated Ki-67 proliferation index (up to 20\%) (Figure 1A-F). Targeted RNAsequencing identified a fusion of MTAP (NM_002451.3) exons 1-7 to BRAF (NM_004333.4) exons 9-18. Resection margins were negative. The patient is disease-free three years post-resection. Case 2, a 12-year-old female with a $5.3 \mathrm{~cm}$ rapidly enlarging heel mass encasing and invading the calcaneus was diagnosed with a juvenile xanthogranuloma (JXG) family lesion (CD163/CD68/CD14/fascin/Factor XIIla+) (Figure 1G-M). Despite the lack of cytologic atypia or increased mitotic rate by $\mathrm{H} \& \mathrm{E}$ stain, a Ki-67 proliferation index stain revealed increased staining in lesional cells up to $40 \%$. Targeted RNA-seq identified a fusion of MS4A6A (NM_022349.3) exons 1-6 and BRAF (NM_004333.4) exons 11-18. During staging, the patient was found to have PET-avid dissemination to lymph nodes and lung (Figure $1 \mathrm{~N}-\mathrm{P}$ ). While the morphologic features were consistent with a low-grade histiocytic lesion of JXG phenotype, the integration of the 1) high Ki-67 proliferation index, 2) aggressive clinical behavior with lymphatic/metastatic-like spread, and 3) novel molecular BRAF-fusion suggested an atypical JXG family neoplasm with uncertain biological behavior. The patient was treated with 12 cycles of clofarabine with clinical remission of metastatic sites and near clinical remission at primary site now 12 months off therapy.

MTAP-BRAF and MS4A6A-BRAF fusions are predicted to contain all functional domains of MTAP and MS4A6A, respectively, along with the BRAF kinase domain but no $\mathrm{N}$-terminal regulatory, RASbinding domain (Figure 1Q). For molecular and therapeutic characterization, MTAP-BRAF and MS4A6A-BRAF were cloned and stably expressed in a heterologous cell model since patientderived cell lines were lacking. The NIH/3T3 cells model system was utilized due to its ability to reliably discern oncogenic fusion profiles ${ }^{9-11}$. In soft agar assays, both MTAP-BRAF and MS4A6A$B R A F$ expressing NIH3T3 showed a significant increase in colony count over control $(p<0.0001$, Figure 1R). Next, we tested activation of downstream MAPK and PI3K/mTOR pathways. Under serum starved conditions, we observed elevated levels of phosphorylated-ERK and -S6 in both BRAF-fusion expressing cells compared to controls, indicating aberrant activation of both the MAPK and PI3K/mTOR pathway, respectively (Figure $1 \mathrm{~S}$ ). Slightly higher PI3K/mTOR pathway activation levels in MTAP-BRAF versus MS4A6A-BRAF cells could be partly explained by higher MTAP-BRAF protein expression (Figure 1S, Myc-tag blot).

A single report on BRAF-fusions in $\mathrm{LCH}^{4}$ has shown unresponsiveness to BRAF-V600E specific inhibitors such as vemurafenib, termed first-generation RAF inhibitors (RAFi), but observed suppression by second-generation RAFi, PLX8394, and downstream MEK inhibition, similar to other pediatric glioma studies on BRAF-fusions ${ }^{9,11}$. Herein, we evaluated the responsiveness of novel MTAP-BRAF and MS4A6A-BRAF to such targeted inhibitors. Upon targeting the NIH3T3 
models with first-generation RAFi PLX4720, as expected, no suppression of BRAF-fusion driven signaling or growth was observed (Supplemental Figure 1A). Interestingly, second-generation RAFi PLX8394 also showed no suppression in MTAP- or MS4A6A-BRAF driven soft agar growth despite targeting MAPK/PI3K_signaling (Figure 2A-B). This is in contrast to PLX8394-mediated suppression of BRAF-fusion driven growth in the previously described $\mathrm{LCH}^{4}$ and other cancers, such as the KIAA1549-BRAF fusion in pediatric glioma ${ }^{9-11}$. PLX8394 suppressed FAM131B-BRAF (a pediatric glioma derived fusion ${ }^{12,13}$ ) and BRAF-V600E driven growth and signaling as well as actively disrupted FAM131B-BRAF dimers (Supplemental Figures 1B-D), highlighting therapeutic differences between MTAP-/MS4A6A-BRAF, BRAF-V600E and other BRAF-fusions.

BRAF-fusions function as active homo- and heterodimers (with wild-type BRAF) to mediate cell signaling ${ }^{9,11}$. We found that MTAP- and MS4A6A-BRAF also mediate such protein-protein interactions in co-immunoprecipitation assays (Figure 2C-D, DMSO lanes). PLX8394 blocks BRAF kinase activity via disrupting BRAF dimerization ${ }^{14}$ but we observed no disruption of MTAP- and MS4A6A-BRAF fusion dimerization with PLX8394 (Figure 2C-D, PLX8394 lanes), thereby providing a plausible explanation for PLX8394 unresponsiveness in soft agar assays though MAPK/PI3K signaling remains discordantly suppressed by some unknown mechanism. This distinct unresponsiveness to pan-RAFi represents a significant departure from the current view that $B R A F$-fusions and other BRAF mutations should respond to second-generation RAFi such as PLX8394 ${ }^{9,15}$. We found that this difference arises due to the contribution of N-terminal partners, MTAP (exons 1-7) and MS4A6A (exons 1-6), to respective fusion dimerization that is unaffected by PLX8394 (Figure 2C-D, lanes 3,7). Similar role of $\mathrm{N}$-terminal partner accounts for differential response of CRAF-fusions to PLX8394 ${ }^{10}$. Furthermore, we observed that Trunc-MTAP (exons 1-7) competitively substituted MTAP-BRAF homo-dimerization in a dose-dependent manner, suggesting preferential and potent protein interactions mediated by $\mathrm{N}$-terminal partner in these histiocytic-specific BRAF-fusions (Figure 2E).

To target dimerization-dependent oncogenicity of MTAP- and MS4A6A-BRAF via a different mechanism, we tested LY3009120, a pan-RAF dimer inhibitor that binds and stabilizes the BRAF dimer in an inactive conformation ${ }^{16}$. LY3009120 showed robust suppression of both fusionmediated signaling and colony transformation (Figure 2B) while stabilizing the MTAP- or MS4A6A-BRAF in inactive conformation (Figure 2C-D, respectively, lanes 9-11). We also tested the effect of FDA-approved MEK inhibitors (MEKi) ${ }^{17}$, selumetinib and trametinib. We observed dose-dependent decrease in phospho-ERK and growth with trametinib (Figure $\underline{2 F}$ ) and selumetinib (Supplemental Figure 2) in both BRAF-fusion models suggesting downstream MEKi as a therapeutic alternative to RAFi.

Though functional data predicts similar effects for these two novel fusions, each neoplasm had discordant clinical and pathologic phenotypes. Malignant histiocytic lesions typically have aggressive behavior ${ }^{18}$, unlike case 1 . Typical JXG family lesions often show indolent behavior in 
pediatric patients ${ }^{19}$. Even in rare systemic presentations, they do not feature a lymphatic-type dissemination, as demonstrated in case 2. Furthermore ${ }_{L}$ Ki-67 proliferation index in JXG lesions is typically less than $20 \%$, and no higher than $10 \%$ (unpublished data, JP). Thus, the focally high proliferation rate (40\%) in the MS4A6A-BRAF JXG family lesion was the first correlate to its aggressive clinical behavior. On the contrary, the MTAP-BRAF malignant histiocytic neoplasm had only modestly elevated proliferation rate (up to $20 \%$ ), which is lower than most malignant histiocytic neoplasms (often $>30 \%$ ) $^{20}$. This may correlate with its more indolent behavior, despite its high-grade cytologic features. Both of these unusual, divergent phenotypes further emphasize that in histiocytic neoplasms, an integrated diagnosis with pathologic, molecular, and clinical/radiographic correlation are all needed for best diagnosis and allows for expanded treatment options.

\section{Acknowledgments:}

The authors would like to thank Dr. Ronald Jaffe for second review of the cases and appraisal of the manuscript.

This work was supported by the Children's Brain Tumor Tissue Consortium funding sources (P.J., A.J.W., P.B.J., and A.C.R).

\section{Authorship Contribution:}

J.P., L.F.S, P.J. conceived the study; J.P., L.F.S and P.R. generated, analyzed and interpreted the pathology data; P.J. and J.S. performed molecular experiments as well as analyzed and interpreted the results; P.J., L.F.S, J.S. and J.P. wrote the manuscript and compiled the figures; P.J., L.F.S, J.S., P.R., R.W., M.L., P.B.S., A.J.W., M.D.H., A.C.R and J.P. edited and reviewed the manuscript.

Conflict-of-interest disclosure: The authors declare no competing financial interests.

Correspondence: Jennifer Picarsic, Department of Pathology, University of Pittsburgh School of Medicine Pittsburgh, PA USA, e-mail: jenpicarsic@gmail.com

\section{$\underline{\text { References: }}$}

1. Diamond EL, Durham BH, Haroche J, et al. Diverse and Targetable Kinase Alterations Drive Histiocytic Neoplasms. Cancer Discov. 2016;6(2):154-165.

2. Durham BH, Diamond EL, Abdel-Wahab O. Histiocytic neoplasms in the era of personalized genomic medicine. Curr Opin Hematol. 2016;23(4):416-425.

3. Ozkaya N, Dogan A, Abdel-Wahab O. Identification and Targeting of Kinase Alterations in Histiocytic Neoplasms. Hematol Oncol Clin North Am. 2017;31(4):705-719.

4. Chakraborty R, Burke TM, Hampton OA, et al. Alternative genetic mechanisms of BRAF activation in Langerhans cell histiocytosis. Blood. 2016;128(21):2533-2537. 
5. Zarnegar S, Durham BH, Khattar $\mathrm{P}$, et al. Novel activating BRAF fusion identifies a recurrent alternative mechanism for ERK activation in pediatric Langerhans cell histiocytosis. Pediatr Blood Cancer. 2018;65(1).

6. Lee LH, Gasilina A, Roychoudhury J, et al. Real-time genomic profiling of histiocytoses identifies early-kinase domain BRAF alterations while improving treatment outcomes. JCI Insight. 2017;2(3):e89473.

7. Durham BH, Lopez Rodrigo E, Picarsic J, et al. Activating mutations in CSF1R and additional receptor tyrosine kinases in histiocytic neoplasms. Nat Med. 2019;25(12):1839-1842.

8. Emile JF, Abla O, Fraitag $\mathrm{S}$, et al. Revised classification of histiocytoses and neoplasms of the macrophage-dendritic cell lineages. Blood. 2016;127(22):2672-2681.

9. Sievert AJ, Lang SS, Boucher KL, et al. Paradoxical activation and RAF inhibitor resistance of BRAF protein kinase fusions characterizing pediatric astrocytomas. Proc Natl Acad Sci U S A. 2013;110(15):59575962.

10. Jain P, Fierst TM, Han HJ, et al. CRAF gene fusions in pediatric low-grade gliomas define a distinct drug response based on dimerization profiles. Oncogene. 2017;36(45):6348-6358.

11. Jain $\mathrm{P}$, Silva $\mathrm{A}$, Han $\mathrm{HJ}$, et al. Overcoming resistance to single-agent therapy for oncogenic BRAF gene fusions via combinatorial targeting of MAPK and PI3K/mTOR signaling pathways. Oncotarget. 2017;8(49):84697-84713.

12. Cin $\mathrm{H}$, Meyer $\mathrm{C}$, Herr $\mathrm{R}$, et al. Oncogenic FAM131B-BRAF fusion resulting from $7 \mathrm{q} 34$ deletion comprises an alternative mechanism of MAPK pathway activation in pilocytic astrocytoma. Acta Neuropathol. 2011;121(6):763-774.

13. Roth JJ, Santi M, Pollock AN, et al. Chromosome band 7q34 deletions resulting in KIAA1549-BRAF and FAM131B-BRAF fusions in pediatric low-grade Gliomas. Brain Pathol. 2015;25(2):182-192.

14. Zhang C, Spevak W, Zhang $Y$, et al. RAF inhibitors that evade paradoxical MAPK pathway activation. Nature. 2015;526(7574):583-586.

15. Okimoto RA, Lin L, Olivas V, et al. Preclinical efficacy of a RAF inhibitor that evades paradoxical MAPK pathway activation in protein kinase BRAF-mutant lung cancer. Proc Natl Acad Sci U S A. 2016;113(47):13456-13461.

16. Peng SB, Henry JR, Kaufman MD, et al. Inhibition of RAF Isoforms and Active Dimers by LY3009120 Leads to Anti-tumor Activities in RAS or BRAF Mutant Cancers. Cancer Cell. 2015;28(3):384-398.

17. Gilmartin AG, Bleam MR, Groy A, et al. GSK1120212 (JTP-74057) is an inhibitor of MEK activity and activation with favorable pharmacokinetic properties for sustained in vivo pathway inhibition. Clin Cancer Res. 2011;17(5):989-1000.

18. Jacobsen E, Abla O, Visser J. Malignant Histiocytoses. In: Abla O, Janka G, eds. Histiocytic Disorders. Cham: Springer International Publishing; 2018:361-381.

19. Ceppi F, Abla O. Juvenile Xanthogranuloma and Related Non-LCH Disorders. In: Abla O, Janka G, eds. Histiocytic Disorders. Cham: Springer International Publishing; 2018:293-311.

20. Picarsic J, Jaffe R. Pathology of Histiocytic Disorders and Neoplasms and Related Disorders. In: Abla O, Janka G, eds. Histiocytic Disorders. Cham: Springer International Publishing; 2018:3-50. 


\section{Figure Legends:}

Figure 1. Novel BRAF-fusions in histiocytic neoplasms mediate oncogenicity via activation of MAPK/PI3K/mTOR pathway: Malignant histiocytic neoplasm with histiocytic and Langerhans cell sarcoma phenotypes with novel MTAP-BRAF fusion, and atypical juvenile xanthogranuloma family lesion with novel MS4A6A-BRAF fusion.

A-F. Case 1 Malignant histiocytic neoplasm with large, pleomorphic cells (A-B) and areas of necrosis $(*)$. Immunohistochemistry with CD163 (C), CD1a (D) and Langerin (E) in a subset of lesional cells. Ki-67 proliferation index (F) was elevated up to $20 \%$, including atypical large cells (F). (Original magnification A. 200x, B, 4000x, C-E. 1000x, F. 200x). BRAF VE1 immunostain was negative (not shown). G-M. Case 2 atypical juvenile xanthogranuloma (JXG) family neoplasm with bland histiocytes $(\mathrm{G}-\mathrm{H})$ and a rare mitosis $(\mathrm{H}$, center). Immunohistochemistry with Factor XIIIa (I) was strongly and diffusely positive. The Ki-67 proliferation index was variable, as high as $40 \%(\mathrm{~J}-\mathrm{K})$ in one core biopsy and as low as $10 \%$ in other core (L-M) taken at the same time and accounting for inflammation, which was low in both core biopsies. (Original magnification: $\mathrm{G}$. 100x, H. 1000x, I. 200x, J. 100x, K. 1000x, L. 100x, M. 1000x). The BRAF VE1 immunostain was negative (not shown). N-P. Case 2 with JXG: Imaging at diagnosis revealed a crescentic enhancing soft tissue mass by magnetic resonance imaging wrapping around the calcaneus, deep to the Achilles tendon ( $N$, arrows) and positron emission tomography (PET) scanning revealed abnormal signal in the ankle (primary), knee, inguinal region and chest (O). Following 9 of 12 cycles of clofarabine, PET scan revealed resolution of disseminated disease and shrinkage of the primary ankle tumor. Q. Structure of novel BRAF-fusions in histiocytic neoplasms. MTAP-BRAF: MTAP exons 1-7 encode phosphate binding sites, trimerization site at Trp189 residue, and substrate binding site, and BRAF exons 9-18 encode the tyrosine kinase domain. MS4A6A-BRAF: MS4A6A exons 1-6 encode 4 transmembrane helical regions, and BRAF exons 11-18 encode the tyrosine kinase domain. R. Soft agar assay using NIH3T3 cells stably expressing BRAF-fusions. Error bars represent $\mathrm{SEM}, \mathrm{n}=5, * * * \mathrm{p}$-value<0.001 compared with control conditions. S. Western blot analysis of MAPK and PI3K/mTOR pathway proteins in NIH3T3 cells stably expressing BRAFfusions. ' $\mathrm{p}$-' and 't-' represent phosphorylated and total versions of protein, respectively.

Figure 2. MTAP-BRAF and MS4A6A-BRAF fusions are not suppressed by second generation RAF inhibitors but demonstrate sensitivity to LY3009120 and MEK inhibitors.

A. Western blot analysis (left) and soft agar colony counts (right) showing the effect of second generation RAFi, PLX8394, on NIH3T3 cells expressing MTAP-BRAF and MS4A6A-BRAF respectively. B. Western blot analysis (left) and soft agar colony counts (right) showing the effect of pan-RAF-dimer inhibitor, LY3009120, on NIH3T3 cells expressing MTAP-BRAF and MS4A6ABRAF respectively. C. Co-immunoprecipitation (co-IP) assay assessing homo-dimerization of MTAP-BRAF as well as hetero-dimerization with wild-type BRAF and Trunc-MTAP in HEK293 cells 
under control, PLX8394, and LY3009120 treated conditions. D. Co-immunoprecipitation assay assessing homo-dimerization of MS4A6A-BRAF as well as hetero-dimerization with wild-type BRAF and Trunc-MS4A6A in HEK293 cells under control, PLX8394, and LY3009120 treated conditions. E. Competition co-IP assay assessing preferential interaction of Trunc. MTAP with MTAP-BRAF fusion versus homo-dimerization. Increasing doses of tetracycline $(0,0.1,0.5,1$ $\mathrm{ug} / \mathrm{ml}$ ) used to regulate protein level of His-tagged Trunc-MTAP. F. Western blot analysis (left) and soft agar colony counts (right) showing the effect of MEK inhibitor, trametinib, on NIH3T3 cells expressing MTAP-BRAF and MS4A6A-BRAF respectively. Error bars represent $S E M, n=3$. No value on bar represents NS (non-significant), ${ }^{*}$-value $<0.05, * * p$-value $<0.01, * * * p$-value<0.001 compared with control conditions. ' $\mathrm{p}$-' and 't-'represent phosphorylated and total versions of protein, respectively. 
Figure 10:Rxiv preprint doi: https://doi.org/10.1101/2020.04.13.039032; this version posted April 14, 2020. The copyright holder for this preprint (which
was not certified by peer review) is the author/funder. All rights reserved. No reuse allowed without permission. Case 1: MTAP-BRAF
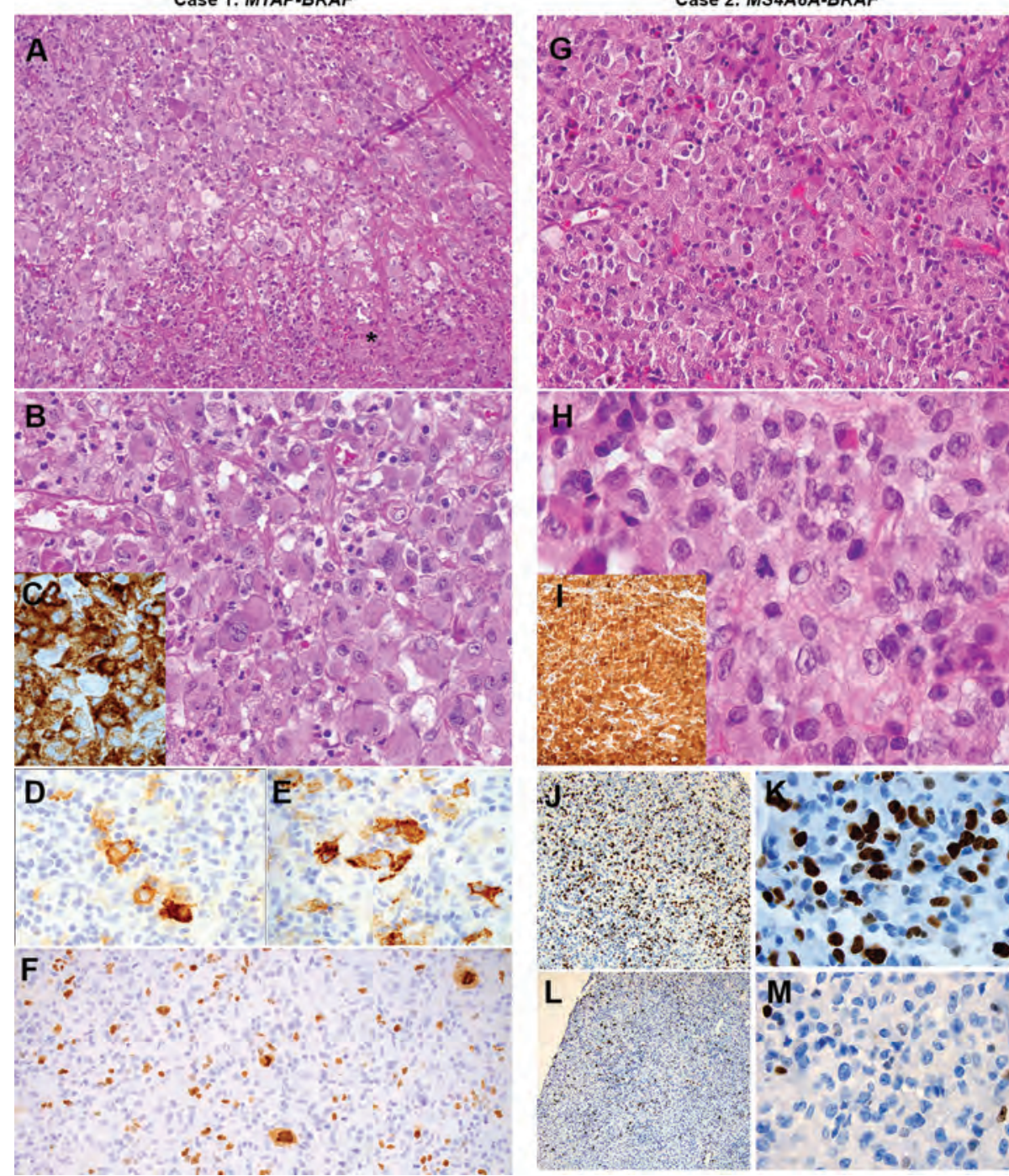
Case 2: MS4A6A-BRAF
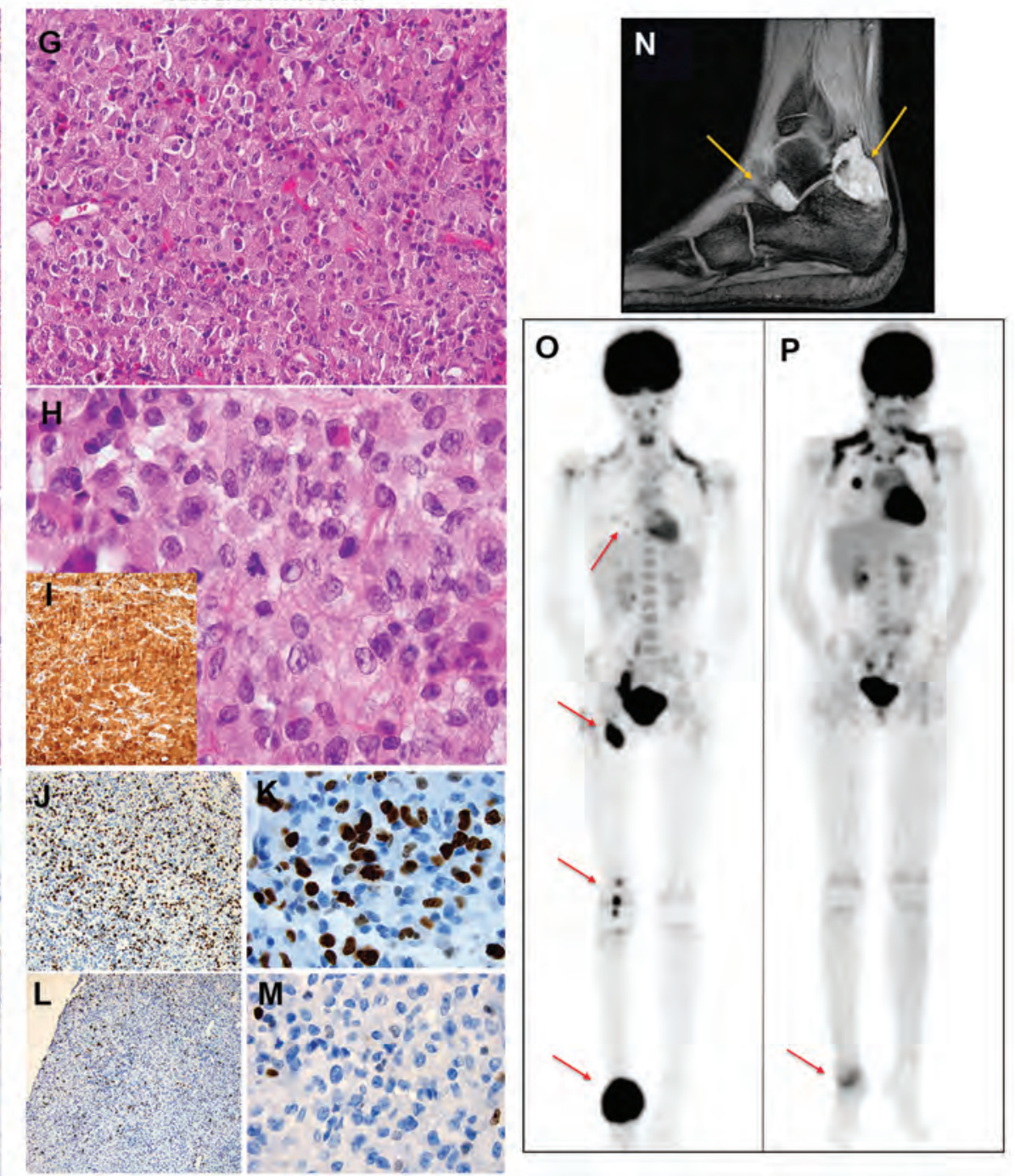

Q.

MTAP-BRAF

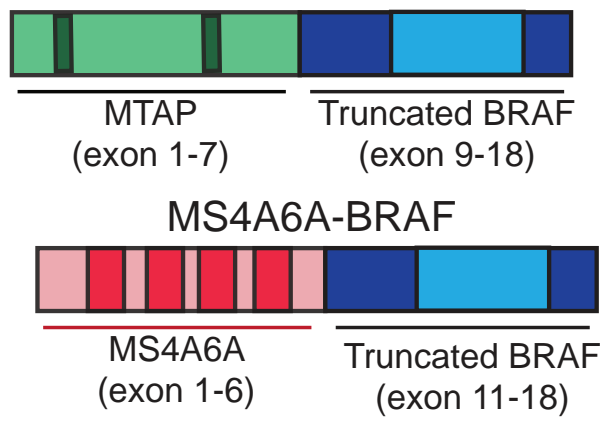

R.

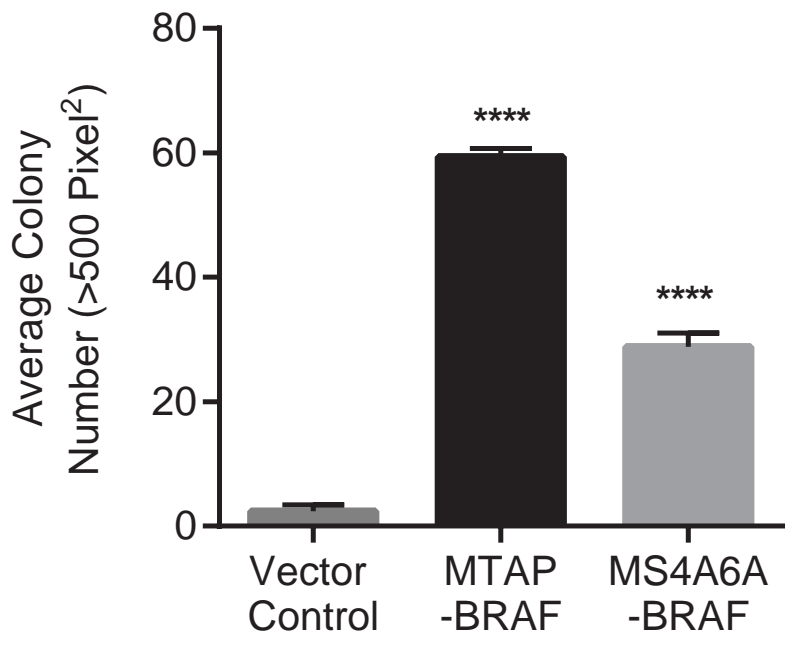

S.
Phosphate

binding site

Protein Kinase

Transmembrane helical region

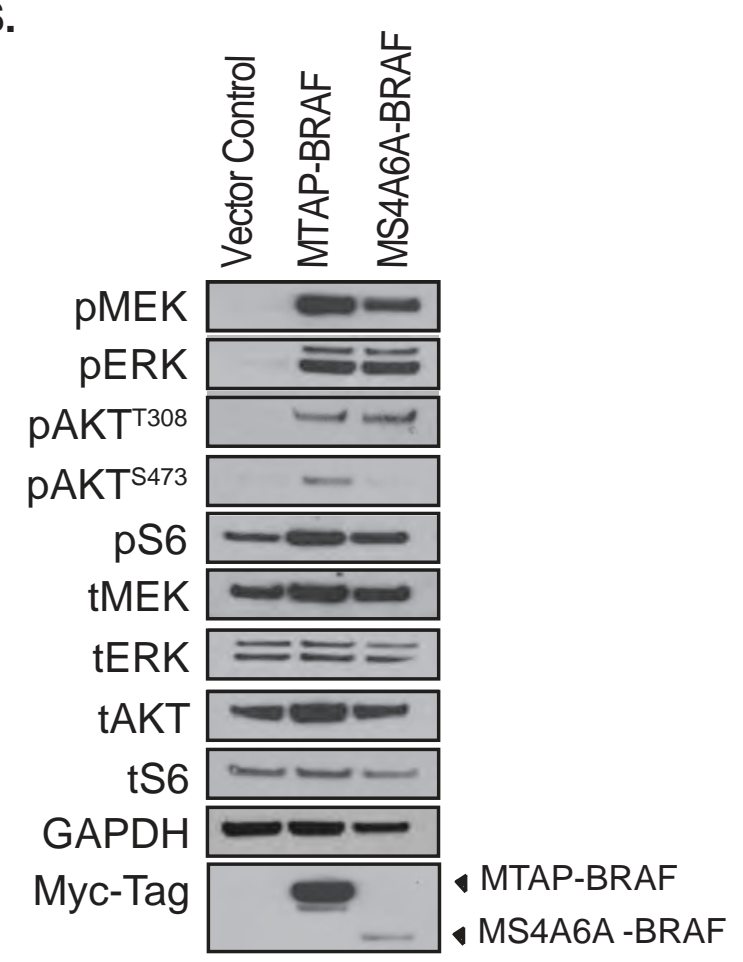


Figure 2 bioRxiv preprint doi: https://doi.org/10.1101/2020.04.13.039032; this version posted April 14, 2020. The copyright holder for this preprint (which

A. was not certified by peer review) is the author/funder. All righ

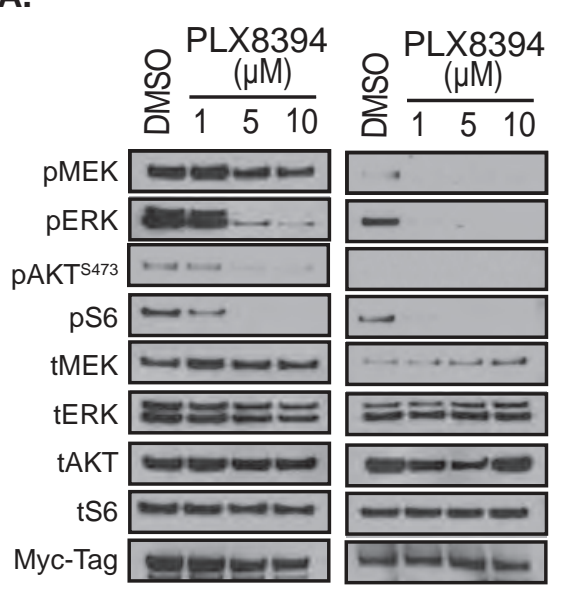

MTAP-BRAF MS4A6A-BRAF

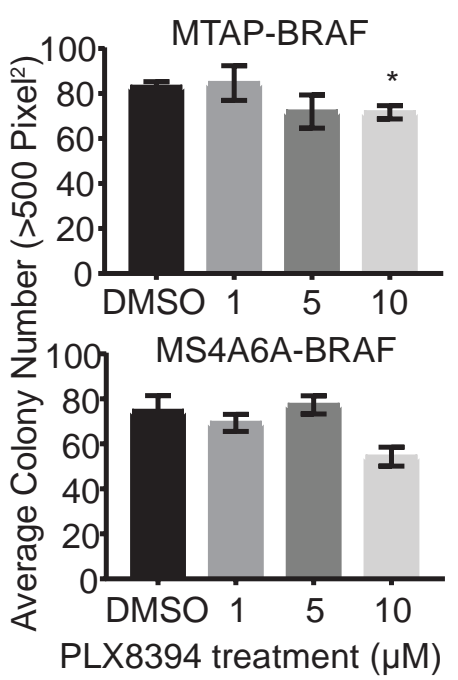

C.

MTAP-BRAF F/M F F M F/M F F M F/M F F M

BRAF - M - - M - - M -

Trunc. MTAP - $-M-\quad-M-1-M$ -

Vector Control - - - F - - - F - - - F

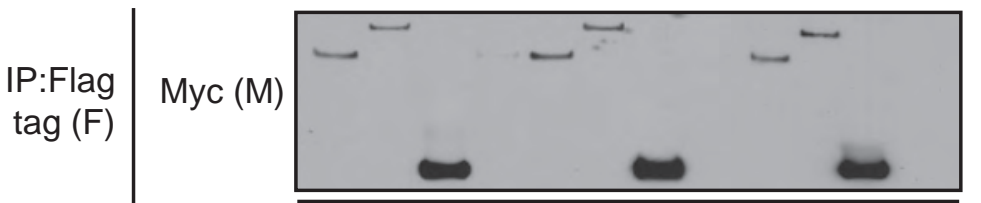

E

Tetracycline

MTAP-BRAF F/M F/M F/M F/M M M M M

$\begin{array}{lllllllll}\text { Trunc. MTAP } & \mathrm{H} & \mathrm{H} & \mathrm{H} & \mathrm{H} & \mathrm{H} & \mathrm{H} & \mathrm{H} & \mathrm{H}\end{array}$

Vector Control - $\quad$ - $\quad$ - $\quad$ - $\quad$ F F F F

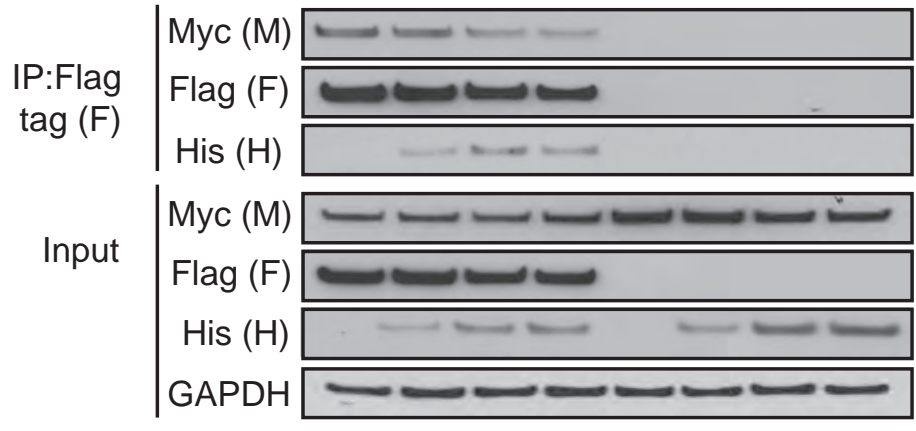

B.

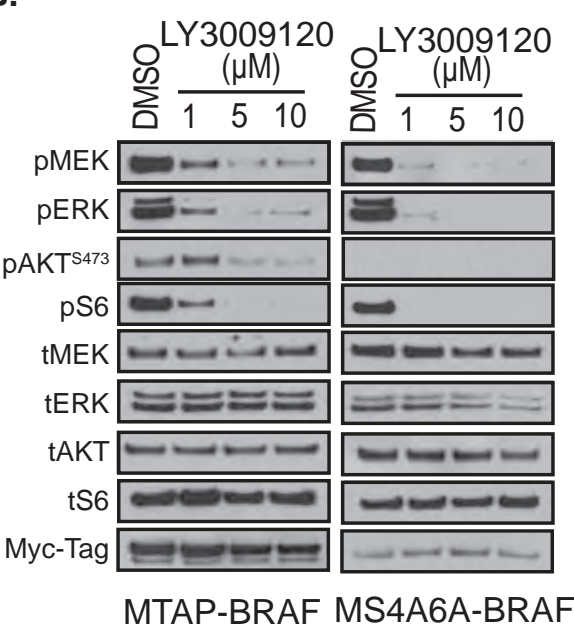

D.

MS4A6A-BRAF F/M F F M F/M F F M F/M F F M

BRAF - M - - M - - M -

Trunc. MS4A6A - - M - - - M - - - M -

Vector Control $\quad-\quad-\quad-F \quad-\quad-\quad-F \quad-\quad-\quad-F$

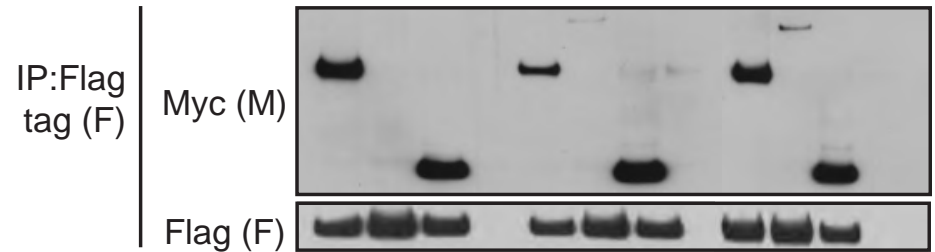

Input

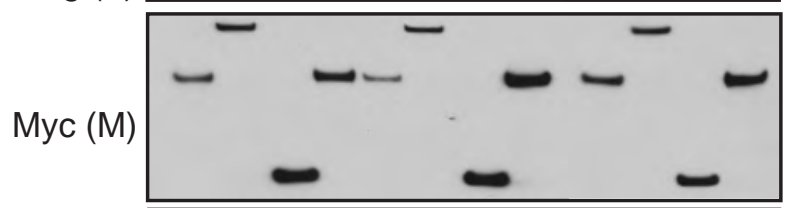

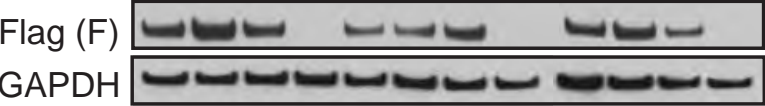

F.

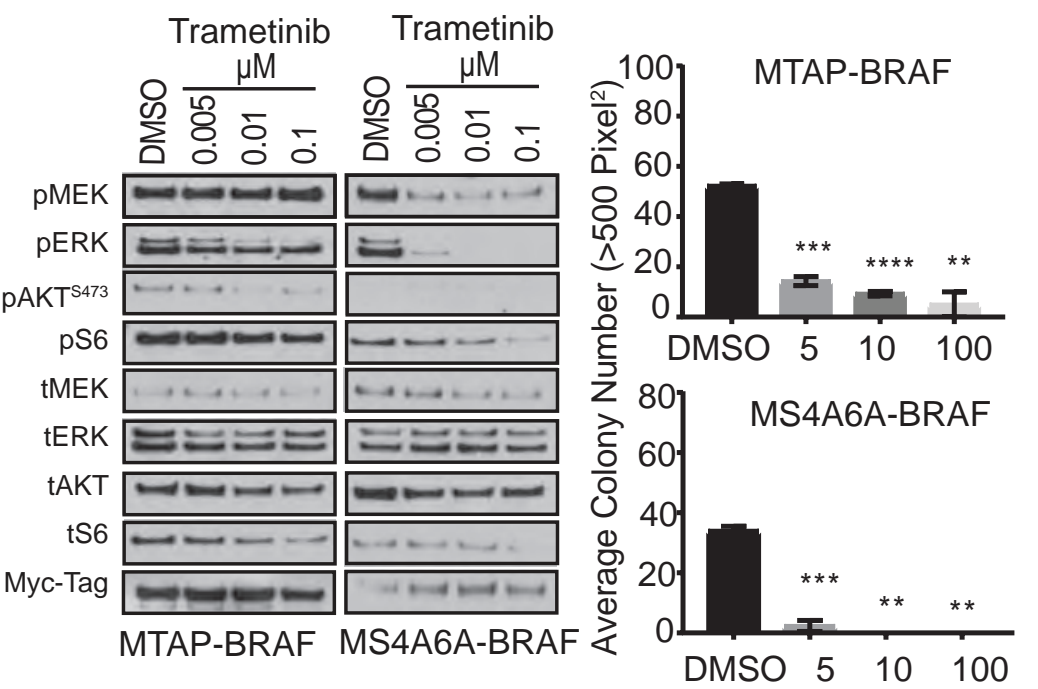

Trametinib treatment (nM) 